\title{
Sleep Quality, Cancer-Related Fatigue, and Health- Related Quality of Life Among Omanis Hospitalized Patients with Cancer: A Cross-Sectional Study.
}

Zamzam Al-Habsi ( $\square$ mdt.breast.squh@gmail.com )

Sultan Qaboos University Hospital

Huda Al-Noumani

Sultan Qaboos University College of Nursing https://orcid.org/0000-0002-8975-3150 Iman Al Hashmi

Sultan Qaboos University College of Nursing

\section{Research Article}

Keywords: Sleep, fatigue, health-related quality of life, predictors, Cancer, Oman

Posted Date: May 20th, 2021

DOl: https://doi.org/10.21203/rs.3.rs-274232/v1

License: (c) (i) This work is licensed under a Creative Commons Attribution 4.0 International License.

Read Full License 


\section{Abstract}

Purpose: This study aimed to examine the difference in HRQoL by participants' characteristics and to investigate the determinants of health-related quality of life HRQoL among Omani hospitalized patients with cancer.

Methods: This cross-sectional study was conducted in two oncology centers in Oman. Omani hospitalized patients with breast, thyroid, colorectal, stomach, and prostate cancer were recruited using convenience sampling. Participants completed Arabic versions of self-reported questionnaires: Pittsburgh Sleep Quality Index, Brief Fatigue Inventory scales and Functional Assessment of Cancer Therapy. Descriptive and inferential statistics were performed. T-test, ANOVA and multiple linear regression analysis was utilized to determine predictors of HRQOL.

Results: in total, 275 participants were recruited (Mean ${ }_{a g e}=52$ years). About $64 \%$ of the participates reported poor sleep and $18.5 \%$ reported severe cancer-related fatigue. Sleep quality, cancer-related fatigue, age, and prostate and thyroid cancer were significant predictors of $\operatorname{HRQoL}(F(5,269)=26.26, p<0.000)$ and they explained $33 \%$ of the variances in the HRQoL $\left(R^{2}=.328\right)$.

Conclusions: This study highlights the impact of sleep quality and caner-related fatigue on the HRQOL among Omanis hospitalized with cancer. Thus, sleep quality and cancer-related fatigue should be assessed routinely during hospitalization of oncology patients with special attention patients' age.

\section{Introduction}

Globally, over the past century, there has been a dramatic increase in cancer incidence and mortality, making it the second leading cause of death after cardiovascular disease [1]. Cancer mortality expanded by $25 \%$ from 1990, with an anticipated frequency of 23.6 million cases annually by 2030 [2]. The global cancer burden estimated to have risen to 18.1 million new cases and 9.6 million deaths in 2018 [3]. Cancer has been reported that one in six women and one in five men will develop cancer during their lifespan [4]. In the Eastern Mediterranean region (including the Middle East), about 555,318 cancer cases were reported in 2012 [5]. The number of cancer cases is estimated to double in nearly 20 years, meaning that the Eastern Mediterranean is expected to rank the highest relative increase in the world [5]. In Oman, $30 \%$ of the mortality rate is attributed to neoplasm; the crude cancer incidence rate among Omanis was 63.91 per 100,000 for men and 74.88 per 100,000 for women [6]. Furthermore, for solid cancer, breast cancer is the most common type of cancer among Omani female (24.48\%), followed by thyroid (15.47\%) and colorectal $(7.51 \%)$. Among Omani males, prostate cancer was the most common solid type (11.08\%), followed by colorectal (10.95\%), and stomach (6.00\%) [6].

Universally, cancer burden and its management have been linked to consequences such as financial burden, increased morbidity, impaired quality of life, and premature death [7]. Cancer impacts on patients' physiological, psychological, and social status have been the subject of concern lately [8]. Sleep quality and cancer-related fatigue are two significant physical consequences experienced by adult patients with 
cancer affecting their quality of life and disabling daily functioning, memory, and concentration [8]. Health-related quality of life (HRQoL) is commonly used to observe the influence of health status on the general quality of life and is used as a primary outcome measure in studies evaluating the success and effectiveness of cancer treatment [9]. Poor sleep quality is known to be a dominant concern in about $60 \%$ of patients with cancer [10] with a potential effect on patients' HRQoL, which has been coupled with a decrease in functional performance and psychological status of patients with cancer [11]. Like poor sleep quality, severe cancer- related fatigue is commonly found among $25 \%$ to $99 \%$ of patients with cancer [12]. Cancer-related fatigue is a common side-effect of cancer and its treatment that is regarded as a risk factor leading to a reduction in cancer survival and HRQoL [12] and treatment suspension [13].

Similar to other Middle Eastern countries, patients' characteristics, sleep quality, and cancer-related fatigue and their influence on HRQoL among patients with cancer have not received adequate attention in Oman. The aims of this study were to examine 1) difference in HRQoL by participants" demographical and clinical characteristics, sleep quality, and cancer-related fatigue, and 2) determinants of HRQoL among hospitalized Omani patients with cancer.

\section{Methods}

A descriptive correlational cross-sectional was carried out in two oncology centers in Oman, which are considered the country's main oncology centers that provide management to all patients with cancer. The study was conducted from June to October 2019.

We used a non-probability convenience sampling to recruit patients with cancer. The sample size was determined based on the numbers of independent variables included in multivariate regression analysis, which was recommended to be 10-20 participants per variable [14]. As a rule of thumb, we counted 15 participants per variable (total of 18 variables). Accordingly, the required sample size was 270 participants. The total participants included in the study were 275 .

Participants were included if they were: Omani aged $\geq 18$ years, hospitalized, diagnosed with cancer (i.e., breast, thyroid, colorectal, stomach, prostate cancer), aware of cancer diagnosis, understand and read Arabic, and willing to participate in the study. Participants who were critically ill, unable to complete the survey, and had cognitive impairment were excluded from the study.

Participants admitted in the oncology, and surgical units were screened for eligibility using a patient list obtained through electronic medical records. Eligible participants were initially contacted by the principal investigator (PI) to inform them about the study purpose and got an informed written consent form. Voluntary participation and refusal to participate would not affect their treatment was assured. Participants self-completed the questionnaire. The clinical data were obtained through medical records by the PI. Data were collected from two main oncology centers, representing 11 governorates of Oman, from June to October 2019. Permission to use all questionnaires described hereinafter was obtained from the primary developer. The study was approved by the Ethics and Research Committees of both centers: 
Sultan Qaboos University and Ministry of Health (CON/EA/26/2019, SRC\#46/2019), respectively. The study was performed in line with the principles of the declaration of Helsinki.

All participants completed the Pittsburgh Sleep Quality Index (PSQI), the Brief Fatigue Inventory (BFI), and Functional Assessment of Cancer Therapy (FACT-G) questionnaires. In this study, sleep quality was assessed by the Pittsburgh Sleep Quality Index (PSQI) [15]. PSQI is a self-report measure with evidence of good internal homogeneity, test-retest reliability, validity, sensitivity (89.6\%), specificity (86.5\%), and Cronbach's alpha of 0.83 [15]. The PSQI distinguishes "poor" from "good" sleep that has been used in the cancer population widely [16] and the Arab cancer population, specifically [17]. PSQI is rated on a 0-3 Likert-like scale, whereby 0 (no difficulty) to 3 (severe difficulty). In a clinical cancer population, a cutoff score of $\geq 8$ suggests a poor sleep, while a score of $\leq 7$ indicates a good sleep quality $[15,18]$. The PSQI Arabic translated version showed internal consistency of 0.74 in the Arabic cancer population [19]. In the current study, Cronbach's alpha of PSQI was 0.63 , and we categorized sleep as poor quality (score $\leq 7$ ) or good quality (score $\geq 8$ ).

Cancer-related fatigue was assessed using the Brief Fatigue Inventory scale (BFI), a reliable tool established for the rapid assessment of fatigue severity in clinical screening for patients with cancer [20]. The scale's items are rated numerically from 0 to 10 and consists of nine items; the first three assess fatigue severity ( $0=$ No fatigue and $10=$ as poor as you can imagine for the first 3 items), while the remaining six items assess fatigue interference with general activity, mood, walking, work, relation and enjoyment $(0=$ Does do not interfere, $10=$ completely interfere). The BFI psychometric evaluation supports its internal consistency and constructs validity with Cronbach's alpha of 0.96 [20]. BFI scoring is categorized into two groups: 1) severe fatigue (score of 7-10) and 2) non-severe fatigue (score of 0-6) with a higher score indicating higher fatigue severity. In the present study, fatigue was categorized as non-severe (score of 0-6) and severe (score of 7-10). The Arabic translated version BFI showed good internal consistency and convergent validity with a Cronbach alpha of 0.86 [18]. In the present study, BFI Cronbach's alpha was 0.89 .

Health-related quality of life (HRQoL) was measured by using version 4 of the FACT-G that was developed by David Cella and to be used in the oncology population [21]. FACT-G has been administered in several types of cancer, validated with reliability Cronbach's alpha of 0.88 , and translated into different languages [22]. FACT-G includes four domains: physical, social and family, emotional, and functional [21]. FACT-G version 4 has 27 items; each answered using a 5-point Likert Scale ranging from 0 (Not at all) to 4 (Very much). Total scale and subscale scores are summed to yield 108 points, with the higher scores indicating better HRQoL [22]. The current study showed a Cronbach's alpha of the FACT-G of 0.84 for the total score.

\section{Data analyses}

Statistical Package for the Social Sciences (SPSS) Version 24 was used for data entry and analysis. In this study, data were tested for normality by using Histogram, Plots, Skewness, and Kurtosis where all data were normally distributed. For the study variables description, continuous variables presented with mean and standard deviation, while categorical variables were presented with frequencies and 
percentages. The categorical association was compared by using the Chi-square test, and continuous association was compared by using the T-test, Pearson correlation, and One-Way Analysis of Variance (ANOVA). To examined the determinants of HRQoL, a multiple linear regression analysis was used. Multiple linear regression assumptions (i.e., normal distribution, linearity, and multicollinearity) were examined and not violated.

\section{Results}

\section{Sample demographical and clinical characteristics}

A total of 275 participants were included in this study, with a mean age of 52 years $(S D=14.1)$. The majority were female $(65.1 \%)$, had chronic diseases $(56 \%)$, and had a family history of cancer $(37 \%)$. The mean total score of FACT-G was $73.0(S D=16.2)$ above the midpoint of 54. Among HRQoL domains, the social and family well-being scored the highest mean (Mean=21.1, $S D=5.3)$, and physical well-being scored the lowest mean (Mean=15.6, $S D=17.0)$. The majority of participants had breast cancer $(41.1 \%)$, were at stage IV (45.1\%), and in the first year of cancer diagnosis (70\%). Out of 275 participants, $64.4 \%$ were anemic and on chemotherapy (43.3\%). The majority (64.4\%) reported poor sleep quality, and $18.5 \%$ reported non-severe cancer-related fatigue (Table 1).

\section{Health-related quality of life by patient's demographical and clinical characteristics, sleep quality, and cancer-related fatigue}

We used Pearson correlation, t-test, and ANOVA analysis to examine the difference in HRQoL by patients' characteristics, sleep quality, and cancer-related fatigue. The findings showed that age was significantly and positively related to HRQoL $(r=0.25, p<0.001)$ reflecting as age increased, HRQoL is getting better. In regard to demographical variables, HRQoL varied significantly by level of education $[\mathrm{F}(3,271)=12.26, p=$ $0.000]$, family history of cancer $[t(273)=2.29, p=0.017]$ and income $[\mathrm{F}(3,271)=8.56, p=0.000]$. In regard to clinical variables, HRQoL varied significantly by type of cancer $[F(4,270)=7.34, p=<0.001]$, stage of cancer $[\mathrm{F}(3,271)=9.55, p=0.000]$, chemotherapy status $[\mathrm{F}(2,272)=7.84, p=0.000]$, and anemia status [t $(275)=3.91, p<0.001]$. HRQoL was also significantly affected by sleep quality [t $(273)=-7.90, p=0.00]$, and cancer-related fatigue $[t(273)=-5.54, p=0.000]$ (Table 2).

\section{Determinants of health-related quality of life}

A multiple linear regression model was used to examine the determinants of HRQoL. Variables that influenced HRQoL significantly (i.e., income, educational status, age, family history of cancer, chronic disease, sleep quality, cancer-related fatigue, cancer stage, cancer type, years of disease diagnosis, anemia status, and chemotherapy status; table 2 ) were only entered into the regression model. The 
multiple linear regression model showed that age, sleep quality, cancer-related fatigue, and cancer type were significant determinants of HRQoL. The model was significant, $(F(5,269)=26.26, p<0.000)$ and it had explained $33 \%$ of the variance in $\mathrm{HRQoL}\left(R^{2}=.328\right)$. The model showed that HRQoL is worse in patients with advanced age $(B=-0.266, p=0.000)$ and patients who had poor sleep quality $(B=-11.387$, $p=0.000)$ and severe cancer-related fatigue $(B=-6.639, p=0.003)$. Furthermore, the model indicated that HRQoL is significantly better in patients with prostate and thyroid cancer $(B=8.466, p=0.001 ; B=6.225$, $p=0.047$ ), respectively. (Table 3).

\section{Discussion}

This study was conducted in Oman. It included 275 participants diagnosed with breast, thyroid, colorectal, stomach, or prostate cancer. The main aim of the study was to examine the determinants of HRQoL in hospitalized patients with cancer in Oman and to investigate if HRQoL varied by patients' demographical and clinical variables, cancer-related fatigue, and sleep quality. Findings of the study showed that HRQoL differed significantly by patients' demographical and clinical characteristics, sleep quality, and cancer-related fatigue. Furthermore, results showed that age, poor sleep quality, severe cancer-related fatigue, and being diagnosed with thyroid or prostate cancer were significant determinants of HRQoL.

Our findings showed that $64 \%$ of Omani patients with cancer reported poor sleep quality, which is similar to a Lebanon study that showed poor sleep quality was high among patients who had non-metastatic breast cancer during active treatment [17]. It is also consistent with findings of a systematic review that reported about $30-70 \%$ of newly diagnosed patients with breast, thyroid, colorectal, stomach, and prostate cancer had poor sleep quality [23]. Various factors suggested the growth of poor sleep quality among patients with cancer, of which, cancer treatments modalities, the frequent awakening of patients in midnight and early morning for a nursing procedure or therapy administration, breath discomfort, hospital environmental noise, pain, and worries about illness and treatment are some examples influencing sleeping patterns [17]. In Oman, these factors could also explain why the majority of patients reported poor sleep quality taking into consideration that about $82 \%$ of patients were receiving chemotherapy. This study established baseline data about sleep quality in patients with cancer in Oman. Our finding highlights that health care professionals should pay attention to patients' sleep patterns and quality and identify specific factors that can be managed to improve patients' sleep quality and, subsequently, improve their health-related quality of life.

The mean total score of HRQoL was $73.0(S D=16.2)$, which was above the midpoint of 54 . Physical wellbeing, which involves issues related to pain, nausea, low energy, feeling ill, treatment's side effects, and trouble meeting family's needs, showed the lowest score among HRQoL domains, and physical well-being seemed to pose the most significant challenge for patients with cancer in Oman. A similar result was found in Saudi Arabia, a middle eastern Arabic country, for patients with solid cancer [24]. A Low physical well-being score could be related to the fact that the majority of the Omani patients included in this study were in stage III and IV, were anemic, and had poor sleep quality, all of which have been found to influence 
the overall HRQoL in current study significantly. Additionally, in this study, social and family well-being domains (feeling like being close to friends, family and friend's support, family cancer acceptance, feeling close and getting enough support and love from the partner) were reported the highest score compared to the other three domains. This finding is parallel to a previous Omani study among oncology population that found Omani patients said that their relationships with husbands and family members became more substantial and more supportive after diagnosis with cancer [25], which could explain our findings considering that $73.8 \%$ of patients were married. This finding also supports the notion that social support can improve distress and alleviate coping skills among Omani women challenged with cancer and its management [26]. This finding denotes that healthcare providers should improve patients' physical wellbeing and correlate this domain to patients' sleep quality.

In this study, we found that poor sleep quality, severe cancer-related fatigue, age, and type of cancer (prostate and thyroid) significantly determine HRQoL and explained about $33 \%$ of the variance in HRQoL. This study had found that those participants with poor sleep quality had decreased HRQoL in general. This finding is in line with the literature that showed sleep quality as one of the main predictors of HRQoL; for instance, $\mathrm{Ha}$ and colleague (2019) reported that sleep quality was an important determinant of HRQoL in patients with lung cancer; and that poor sleep quality, dyspnea, fatigue and depression were related to lower HRQoL, and together accounted for $85 \%$ of variances in HRQoL [27]. Likewise, a Nigerian study among women with breast cancer with lower sleep quality showed a significant reduction in HRQoL [28]. In the Arab world, a study in Palestine reported that sleep quality was one of the HRQoL determinants and, together with the pain, were responsible for $42 \%$ of the variance in HRQoL [29]. The positive and significant relationship between poor sleep quality and low HRQoL could be related to the negative impact of poor sleep on the patients' immune systems, making them more susceptible to infection and illness [30]. Another explanation could be linked to the fact that poor sleep tempts changes in the cognitive performance of the patients with cancer, which, as a result, influence their HRQoL [31]. Besides, Poor sleep quality is connected with poor physical well-being, such as gastrointestinal dysfunction that aggravates sickness [32]. The current study extends the observations of the relationship between sleep quality and HRQoL from Western societies to the developing world, specifically the Arabs Omani population, and enlightens future focus to promote screening for and improving sleep quality to enhance HRQoL community with cancer.

Likewise, cancer-related fatigue was found as another determinant of HRQoL in Oman, in which those with severe fatigue had a significant reduction in their overall HRQoL. Our finding is similar to studies from Nigeria, Greek, and France that were conducted in patients with breast and colorectal cancer and found that cancer-related fatigue was a significant determinant of HRQoL [28, 33, 34]. In the Arab population, we couldn't identify literature examining the relationship between fatigue and HRQoL. Many reasons could explain the relationship between fatigue and HRQoL; one reason could be attributed to chemotherapy management and its side-effects [35], and this could explain our finding as about $43 \%$ of our participants were on chemotherapy. Other reasons could be due to the effect of cancer-related fatigue on patients' self-care behavior as they demonstrated less involvement in taking care of themselves, which as a result, affect their HRQoL [36]. Although cancer-related fatigue experienced by the participants in the 
current study was as low as $18.5 \%$, nevertheless, cancer-related fatigue remains a disabling symptom, a common determinant of patients' HRQoL, and it may prolong to two years after diagnosis [33, 34]. These findings could inform the future direction to establish strategies to understand cancer-related fatigue and its attributing factors to enhance HRQoL of patients with cancer.

In this study, age was also found to determine HRQoL in the Omani oncology population, indicating that HRQoL is better among younger age. Our findings were similar to results among cancer population from Sweden and Turkey [37,38], but inconsistent with a study in Yemen, an Arab Country, that reported no relationship [39]. This finding could be because as people get older, they demonstrated lower performance in activities of daily living, lower functional abilities, and more risk of depression, which in consequence, reduce the HRQoL and supporting our finding $[37,40]$. Our finding underlines the mounting need to focus HRQoL at a younger age and pay specific attention toward senior patients. In the Omani context of Oman and based on our findings related to the mean age of our participants (52 years), special attention toward improving HRQoL among older patients with cancer should be accentuated.

Thyroid and prostate cancer found to determine HRQoL in hospitalized Omani, and that those with thyroid and prostate cancer reported better HRQoL compared to other types of cancer. Our findings were similar to a study from Iran [41] but inconsistent with studies from Turkey and France who reported no association $[42,43]$. The high survival rate of prostate and thyroid cancer compared to another type of cancer could explain the link between these types of cancer and HRQoL compared to other cancer such as stomach (70\%) and colorectal (80\%) [44].

This study should be considered within the following limitation; first, use of a cross-sectional design limits the ability to establish causal relationships. Second, we use a convenient nonprobability sample, which could impede the generalizability of the findings. However, a heterogeneous sample from the largest two tertiary health settings in Oman could minimize this threat. Third, Pittsburgh Sleep Quality Index (PSQI) instrument had below the satisfactory level of internal reliability (Cronbach's alpha $=.67$ ), which is similar to another recent Omani study that used PSQI among myocardial infarction patients that reported a Cronbach's a of 0.64 [45]. However, having an adequate sample size could provide robust statistical power and confidence in the results.

Sleep quality, cancer-related fatigue, and HRQoL deserve adequate medical attention regarding supportive care and routine assessment. Prompt recognition, observation, and documentation of these variables will guide the clinical teams to develop interventions to improve fatigue and sleep quality and their impact on the health-related quality of life. Holistic multidisciplinary cancer care is necessary to implement by clinicians inside the hospital and out of the hospital to improve all aspects of HRQoL of oncology population. On the other hand, an individualized approach should be maintained considering unique patients' characteristics such as age, cancer type, stage, and treatment modalities. Policymakers should design the oncology centers to empower patients' physical, functional, social, and emotional well-being. Sleep quality, cancer-related fatigue, and HRQoL can be regarded as quality indicators for patients with cancer. Finally, future studies should examine 1) strategies to improve sleep quality and cancer-related 
fatigue across the cancer population in general and the Arab community in specific, 2) the same variables among patients with non-solid cancer like leukemia and 3) determinants of each subscale as this was not the scope of the current study.

\section{Conclusion}

Health-related quality of life is a multidimensional construct that necessitates further understanding. Sleep quality, cancer-related fatigue, and patients' clinical and demographical characteristics could impose critical determination of overall HRQoL.

\section{Declarations}

\section{Funding}

No funding was received for conducting this study

\section{Conflicts of interests}

The author(s) declared no potential conflict of interest with respect to the research, authorship, and/or publication of this article.

\section{Ethics approval}

The study was approved by the Ethics and Research Committees of both centers: Sultan Qaboos University and Ministry of Health (CON/EA/26/2019, SRC\#46/2019), respectively. The study was performed in line with the principles of the declaration of Helsinki and was reported based on STROBE reporting guidelines for cross-sectional studies.

\section{Consent to participate}

Written informed consent was obtained for each participant according to national and institutional guidelines.

\section{Consent to Publish}

NA

\section{Data Availability}

The data set used and/or analyzed during the current study are available from the corresponding author on reasonable request.

\section{Authors' contribution}


All authors contributed to the study conception and design. Material preparation, data collection and analysis were performed by Zamzam Al-Habsi. The first draft of the manuscript was written by Zamzam Al-Habsi, and all authors commented on previous versions of the manuscript. All authors read and approved the final manuscript.

\section{References}

1. World Health Organization (2020) cancer Today. http://gco.iarc.fr/today/home

2. Hamadeh, R. R., Borgan, S. M., \& Sibai AM (2017) Cancer Research in the Arab World: A review of publications from seven countries between 2000-2013. , Sultan Qaboos Univ Med J 2:147-154. https://doi.org/10.18295/squmj.2016.17.02.003

3. World Health Organization (2018) Latest global cancer data. Latest global cancer data

4. American Cancer Society. (2017) Cancer facts and Figures 2017

5. World Health Organization (2017) Early detection of cancers common in the Eastern Mediterranean Region

6. Ministry of Health (2018) Oman Cancer Registry

7. Fitzmaurice C, Dicker D, Pain A, et al (2015) The Global Burden of Cancer 2013. JAMA Oncol 1:505527. https://doi.org/10.1001/jamaoncol.2015.0735

8. Costa DSJ, Mercieca-Bebber R, Rutherford C, et al (2016) The Impact of Cancer on Psychological and Social Outcomes. Aust Psychol 51:89-99. https://doi.org/10.1111/ap.12165

9. Yin S, Njai R, Barker L, et al (2016) Summarizing health-related quality of life (HRQOL): Development and testing of a one-factor model. Popul Health Metr 14:1-9. https://doi.org/10.1186/s12963-0160091-3

10. Mercadante S, Aielli F, Adile C, et al (2015) Sleep Disturbances in Patients with Advanced Cancer in Different Palliative Care Settings. J Pain Symptom Manage 25:1301-1306. https://doi.org/10.1016/j.jpainsymman.2015.06.018

11. Chen D, Yin Z, Fang B (2018) Measurements and status of sleep quality in patients with cancers. Support Care Cancer 26:405-415. https://doi.org/10.1007/s00520-017-3927-x

12. Bower JE (2014) Cancer-related fatigue-mechanisms, risk factors, and treatments. Nat Rev Clin Oncol 11:597-609. https://doi.org/10.1038/nrclinonc.2014.127

13. Wang XS, Woodruff JF (2015) Cancer-related and treatment-related fatigue. Gynecol Oncol 136:446452. https://doi.org/10.1016/j.ygyno.2014.10.013

14. Bujang MA, Sa'at N, Sidik TMITAB (2017) Determination of minimum sample size requirement for multiple linear regression and analysis of covariance based on experimental and non-experimental studies. Epidemiol Biostat Public Heal. https://doi.org/10.2427/12117

15. Buysse DJ, Reynolds CF, Monk TH, et al (1989) The Pittsburgh sleep quality index: A new instrument for psychiatric practice and research. Psychiatry Res 28:193-213. https://doi.org/10.1016/01651781(89)90047-4 
16. Akman T, Yavuzsen T, Sevgen Z, et al (2015) Evaluation of sleep disorders in cancer patients based on Pittsburgh Sleep Quality Index. Eur J Cancer Care (Engl) 24:553-559. https://doi.org/10.1111/ecc.12296

17. Fakih, Rahal M, Hilal L, et al (2018) Prevalence and severity of sleep disturbances among patients with early breast cancer. Indian J Palliat Care 24:35-38. https://doi.org/10.4103/IJPC.IJPC_137_17

18. Suleiman K, Al Kalaldeh M, AbuSharour L, et al (2019) Validation study of the Arabic version of the Brief Fatigue Inventory (BFI-A). East Mediterr Heal J 25:784-790. https://doi.org/10.26719/emhj.19.032

19. Carpenter JS, Andrykowski MA (1998) Psychometric evaluation of the Pittsburgh Sleep Quality Index. J Psychosom Res 45:5-13. https://doi.org/10.1016/S0022-3999(97)00298-5

20. Mendoza TR, Wang XS, Cleeland CS, et al (1999) The rapid assessment of fatigue severity in cancer patients: Use of the brief fatigue inventory. Cancer 85:1186-1196. https://doi.org/10.1002/(SICI)1097-0142(19990301)85:5<1186::AID-CNCR24>3.0.C0;2-N

21. Cella BDF, Tulsky DS, Gray G, et al (1993) The Functional Assessment of Cancer Therapy scale: Development and Validation of the General Measure. Am Soc Clin Oncol 11:570-579

22. Victorson D, Barocas J, Song J, Cella D (2008) Reliability across studies from the functional assessment of cancer therapy-general (FACT-G) and its subscales: A reliability generalization. Qual Life Res 17:1137-1146. https://doi.org/10.1007/s11136-008-9398-2

23. Ancoli-Israel S (2015) Sleep Disturbances in Cancer: A Review. Korean Soc Sleep Med 6:45-49

24. Ahmed AE, Almuzaini AS, Alsadhan MA, et al (2018) Health-Related Predictors of Quality of Life in Cancer Patients in Saudi Arabia. J Cancer Educ 33:1198-1203. https://doi.org/10.1007/s13187-0171198-3

25. Al-Azri M, Al-Awisi H, Al-Rasbi S, Al-Moundhri M (2014) Coping with a diagnosis of breast cancer among Omani women. J Health Psychol 19:836-846. https://doi.org/10.1177/1359105313479813

26. Shams N, Al-azri M (2019) The Effect of Cognitive Behavior Stress Management Program on the Distress, Coping Skills, and the Social Support of Omani Women with Breast Cancer: A Pilot Study. Am Sci Res J Eng Technol Sci 55:170-187

27. Ha D, Ries AL, Swigris JJ (2019) Determinants of Cancer-specific Quality of Life in Veteran Lung Cancer Survivors Eligible for Long-Term Cure. In: bioRxiv

28. Jaiyesimi AO, Sofela EA, Rufai AA (2007) Health related quality of life and its determinants in Nigerian breast cancer patients. Afr J Med Med Sci 36:259-265

29. Dreidi MM, Hamdan-Mansour AM (2016) Pain, Sleep Disturbance, and Quality of Life Among Palestinian Patients Diagnosed with Cancer. J Cancer Educ 31:796-803. https://doi.org/10.1007/s13187-015-0946-5

30. Besedovsky L, Lange T, Haack M (2019) The sleep-immune crosstalk in health and disease. Physiol Rev 99:1325-1380. https://doi.org/10.1152/physrev.00010.2018 
31. Alhola P, Polo-Kantola P (2007) Sleep deprivation: Impact on cognitive performance. Neuropsychiatr Dis Treat 3:553-567

32. Zhao W, Jin H, Xu M, et al (2018) Sleep Quality of Functional Gastrointestinal Disorder Patients in Class-Three Hospitals: A Cross-Sectional Study in Tianjin, China. Biomed Res Int 2018:1-5. https://doi.org/10.1155/2018/3619748

33. Faury S, Rullier E, Denost Q, Quintard B (2020) Quality of life and fatigue among colorectal cancer survivors according to stoma status - the national VICAN survey. J Psychosoc Oncol 38:89-102. https://doi.org/10.1080/07347332.2019.1638481

34. Lavdaniti M, Owens D, Liamopoulou P, et al (2019) Factors Influencing Quality of Life in Breast Cancer Patients Six Months after the Completion of Chemotherapy. Diseases 7:26. https://doi.org/10.3390/diseases7010026

35. Abu Obead K, Yaser S, Khattab M, et al (2014) Chemotherapy-induced fatigue among jordanian cancer patients: What are the contributing factors? Middle East J Cancer 5:75-82

36. O'Regan P, McCarthy G, O'Reilly S, et al (2019) Cancer-related fatigue and self-care agency: A multicentre survey of patients receiving chemotherapy. J Clin Nurs 28:4424-4433. https://doi.org/10.1111/jocn.15026

37. Klompstra L, Ekdahl AW, Krevers B, et al (2019) Factors related to health-related quality of life in older people with multimorbidity and high health care consumption over a two-year period. BMC Geriatr 19:187. https://doi.org/10.1186/s12877-019-1194-z

38. Ogce F, Ozkan S, Baltalarli B (2007) Psychosocial stressors, social support and socio-demographic variables as determinants of quality of life of Turkish breast cancer patients. Asian Pacific $\mathrm{J}$ Cancer Prev 8:77-82

39. Sawsan Ba-Khubaira WA-K (2012) Age related quality of life among selected breast cancer patients in Aden, Yemen. Pan Arab J Oncol 5:14-17

40. Pergolotti M, Deal AM, Williams GR, et al (2017) Activities, function, and health-related quality of life (HRQOL) of older adults with cancer. J Geriatr Oncol 8:249-254.

https://doi.org/10.1016/j.jgo.2017.02.009

41. Heydarnejad MS, Hassanpour Dehkordi A, Solati Dehkordi K (2011) Factors affecting quality of life in cancer patients undergoing chemotherapy. Afr Health Sci 11:266-270

42. Akin S, Guner CK (2017) Determinants of Fatigue, Self-efficacy , and Quality of Life of Cancer Patients During Chemotherapy: A Study from Turkey. J Nurs Sci 3:17-26

43. Metallo M, Groza L, Brunaud L, et al (2016) Long-Term Quality of Life and Pregnancy Outcomes of Differentiated Thyroid Cancer Survivors Treated by Total Thyroidectomy and I131 during Adolescence and Young Adulthood. Int J Endocrinol. https://doi.org/10.1155/2016/7586482

44. American Cancer Society (2020) Survival Rates for Prostate Cancer.

https://www.cancer.org/cancer/prostate-cancer/detection-diagnosis-staging/survival-rates.html

45. Almamari RS, Lazarus ER, Muliira JK (2019) Information needs of post myocardial infarction patients in Oman. Clin Epidemiol Glob Heal 7:629-633. https://doi.org/10.1016/j.cegh.2019.02.006 


\section{Tables}

Table 1: Sample demographical and clinical characteristics and Health-related Quality of Life $(n=275)$ 


\begin{tabular}{|c|c|}
\hline Characteristics & Mean (SD) \\
\hline Age in years & $52.1(14.1)$ \\
\hline \multicolumn{2}{|l|}{ Admission Days in the Hospital } \\
\hline \multicolumn{2}{|l|}{ Anemic Status } \\
\hline Anemic & $177(64.4)$ \\
\hline Non-anemic & $98(35.6)$ \\
\hline Total FACT-G & $73.0(16.2)$ \\
\hline Physical well-being & $15.6(17.0)$ \\
\hline Social/Family well-being & $21.1(5.3)$ \\
\hline Emotional Well-being & $18.0(4.2)$ \\
\hline Functional Well-being & $18.3(5.8)$ \\
\hline Characteristics & $n(\%)$ \\
\hline \multicolumn{2}{|l|}{ Gender } \\
\hline Male & $96(34.9)$ \\
\hline Female & $179(56.1)$ \\
\hline \multicolumn{2}{|l|}{ Level of Education } \\
\hline No education & $83(30.2)$ \\
\hline Basic education & $78(28.4)$ \\
\hline High school & $69(25.1)$ \\
\hline University/College education & $45(16.1)$ \\
\hline \multicolumn{2}{|l|}{ Occupation } \\
\hline Employed & $98(35.6)$ \\
\hline Self-employed & $3(1.1)$ \\
\hline Retired & $43(15.6)$ \\
\hline Searching for job & $2(0.7)$ \\
\hline Student & $1(0.4)$ \\
\hline Social security & $24(8.7)$ \\
\hline Housewife & $104(37.8)$ \\
\hline Marital Status & \\
\hline
\end{tabular}

Page 14/18 


\begin{tabular}{|ll|}
\hline Single & $28(10.2)$ \\
\hline Married & $203(73.8)$ \\
\hline Widow & $10(3.6)$ \\
\hline Monthly income & $34(12.4)$ \\
\hline$\leq 300$ OMR & $137(49.8)$ \\
\hline $301-700$ OMR & $56(20.4)$ \\
\hline 701-1000 OMR & $40(14.5)$ \\
\hline$>1000$ OMR & $41(14.9)$ \\
\hline Presence of chronic disease & \\
\hline Yes & $153(55.6)$ \\
\hline No & $120(43.6)$ \\
\hline Family History of Cancer & \\
\hline Yes & $102(37.1)$ \\
\hline No & $171(62.2)$ \\
\hline Type of Cancer & $192(69.8)$ \\
\hline Breast & $34(12.4)$ \\
\hline Thyroid & $68(24.7)$ \\
\hline Colorectal & $113(41.1)$ \\
\hline Stomach & $23(8.4)$ \\
\hline Prostate & \\
\hline Stage of Cancer & \\
\hline Stage I & \\
\hline Stage II & \\
\hline Stage III & \\
\hline Stage IV & \\
\hline Stage of Cancer & \\
\hline Years since Cancer Diagnosis & \\
\hline One year & \\
\hline
\end{tabular}

Page 15/18 


\begin{tabular}{|ll|}
\hline Two year & $52(18.9)$ \\
\hline Four year & $17(6.2)$ \\
\hline Chemotherapy Status & $14(5.1)$ \\
\hline Received & $107(38.9)$ \\
\hline On Chemotherapy & $119(43.3)$ \\
\hline Not Received & $49(17.8)$ \\
\hline Surgical Status & \\
\hline Done & $191(69.5)$ \\
\hline Not done & $84(30.5)$ \\
\hline Radiotherapy Status & \\
\hline Done & $82(29.8)$ \\
\hline Not done & $193(70.2)$ \\
\hline Immunotherapy Status & \\
\hline On immunotherapy & $86(31.3)$ \\
\hline Received & $27(9.8)$ \\
\hline Not for immunotherapy & $162(58.9)$ \\
\hline Sleep Quality & \\
\hline Poor sleep quality & $177(64.4)$ \\
\hline Good sleep quality & \\
\hline Cancer-related Fatigue & \\
\hline Severe & \\
\hline Non-severe & \\
\hline
\end{tabular}

$\mathrm{SD}=$ Standard deviation; OMR: Omani Riyals; FACT-G: Functional Assessment of Cancer Therapyquestionnaire.

Table 2: Health-related quality of life by patient's demographical and clinical variables 


\begin{tabular}{|c|c|c|c|}
\hline Variables & $n$ & Mean $(S D)$ & $P$-value \\
\hline Age $(r) \dagger$ & 275 & $0.25 \ddagger$ & $<0.001$ \\
\hline \multicolumn{4}{|l|}{ Family history } \\
\hline Yes & 104 & 75.90 (17.23) & 0.03 \\
\hline No & 171 & 71.31 (15.38) & \\
\hline \multicolumn{4}{|l|}{ Level of education } \\
\hline No formal education & 83 & $68.12(16.06)$ & $<0.001$ \\
\hline Basic & 78 & $69.86(15.77)$ & \\
\hline Higher school & 69 & 75.68 (16.36) & \\
\hline University/College & 45 & 83.60 (11.03) & \\
\hline \multicolumn{4}{|l|}{ Income } \\
\hline$\leq 300$ & 138 & $69.70(16.18)$ & $<0.001$ \\
\hline $301-700$ & 56 & $75.75(17.13)$ & \\
\hline $701-1000$ & 40 & $71.23(14.10)$ & \\
\hline$>1000$ & 41 & 82.39 (12.95) & \\
\hline \multicolumn{4}{|l|}{ Family history } \\
\hline Yes & 104 & $75.90(17.23)$ & 0.03 \\
\hline No & 171 & $71.31(15.38)$ & \\
\hline \multicolumn{4}{|l|}{ Type of cancer } \\
\hline Breast & 113 & 72.58 (17.89) & $<0.001$ \\
\hline Thyroid & 26 & 86.65 (11.34) & \\
\hline Colorectal & 68 & $68.70(14.22)$ & \\
\hline Stomach & 37 & $68.22(14.55)$ & \\
\hline Prostate & 34 & 79.32 (11.71) & \\
\hline \multicolumn{4}{|l|}{ Caner stage } \\
\hline Stage I & 6 & $94.83(12.32)$ & $<0.001$ \\
\hline Stage II & 56 & $80.34(15.01)$ & \\
\hline Stage III & 89 & $72.16(16.58)$ & \\
\hline Stage IV & 124 & 69.33 (14.87) & \\
\hline
\end{tabular}




\begin{tabular}{|lccc|} 
Anemic & 113 & $77.50(16.95)$ & $<0.001$ \\
Non-anemic & 162 & $69.93(14.98)$ & \\
\hline $\begin{array}{l}\text { Sleep Quality } \\
\text { Good sleep quality }\end{array}$ & 98 & $83.55(14.28)$ & 0.000 \\
Poor sleep quality & 177 & $69.29(14.33)$ & \\
Cancer-Related Fatigue & & & \\
Non-Severe & 224 & $76.81(14.93)$ & 0.000 \\
Severe & 51 & $63.65(15.39)$ & \\
\hline
\end{tabular}

Table reflects significant values only, † Pearson Correlation, $\ddagger \mathrm{r}$ value

Table 3: Determinants of Health-Related Quality of Life†

\begin{tabular}{|lllllll|}
\hline Variable & $\boldsymbol{B}$ & $\mathrm{SE}(\boldsymbol{B})$ & Beta & $\boldsymbol{t}$ & $\boldsymbol{p}$-value & $95 \% \mathrm{Cl}$ \\
\hline Poor Sleep Quality & -11.387 & 1.817 & -0.337 & -6.265 & 0.000 & $-14.965--7.809$ \\
\hline Age & -0.266 & 0.061 & -0.232 & -4.356 & 0.000 & $-0.387--0.146$ \\
\hline Prostate Cancer & 8.466 & 2.540 & 0.172 & 3.334 & 0.001 & $3.466-13.466$ \\
\hline Severe CRF & -6.639 & 2.193 & -0.159 & -3.027 & 0.003 & $-10.957--2.321$ \\
\hline Thyroid Cancer & 6.225 & 3.123 & 0.106 & 1.994 & 0.047 & $0.077-12.373$ \\
\hline
\end{tabular}

$R^{2}=0.328 ; C R F=$ Cancer-Related Fatigue.

tThis model used a stepwise elimination method. The model included variables: age, educational status, income, chronic disease, family history of cancer, cancer type, cancer stage, years of disease diagnosis, anemia status, chemotherapy status, sleep quality, and CRF.

\section{Supplementary Files}

This is a list of supplementary files associated with this preprint. Click to download.

- STROBE.docx 